K. Miyake

Nagoya Math. J.

Vol. 96 (1984), 83-94

\title{
A GENERALIZATION OF HILBERT'S THEOREM 94
}

\author{
KATSUYA MIYAKE
}

\section{§1. Introduction}

Let $k$ be an algebraic number field of finite degree. We denote the absolute class field of $k$ by $\tilde{k}$, and the absolute ideal class group of $k$ by $C \ell(k)$.

For an unramified abelian extension $K / k$, let $P_{k}(K)$ be the subgroup of $C \ell(k)$ consisting of the all classes the ideals of which become principal in $K$, and $S_{k}(K)$ be the subfield of $\tilde{k}$ corresponding to $P_{k}(K)$ by class field theory. The collection

$$
\left\{S_{k}(K) \mid K \text { is an intermediate field of } \tilde{k} / k .\right\}
$$

stands for the solution for the problem on capitulation of ideals of $k$. Its members seem rather special among intermediate fields of $\tilde{k} / k$, but little is known about their number theoretical characterization.

Our concern in this paper is the degree $\left[\tilde{k}: S_{k}(K)\right]$ which is equal to the order $\left|P_{k}(K)\right|$. The following theorems are classical:

HILBERT's THEOREM 94. If $K / k$ is an unramified cyclic extension, then $[K: k]$ divides $\left|P_{k}(K)\right|$.

The Principal Ideal Theorem. $P_{k}(\tilde{k})=C \ell(k), S_{k}(\tilde{k})=k$, and $\left|P_{k}(\tilde{k})\right|$ $=[\tilde{k}: k]$.

This theorem has been generalized as follows (cf. [3, Theorems 5 and 7]):

THEOREM. Let $\tilde{k}$ be the second class field of $k$, that is, the absolute class field of $\tilde{k}$. Let $\varphi$ be an endomorphism of $\mathrm{Gal}(\tilde{k} / k)$, and $K(\varphi)$ be the subfield of $\tilde{k}$ corresponding to the subgroup

$$
\left\langle g^{-1} \cdot \varphi(g) \mid g \in \operatorname{Gal}(\tilde{k} / k)\right\rangle \cdot \operatorname{Gal}(\tilde{k} / \tilde{k}) .
$$

Then the degree $[K(\varphi): k]$ divides $\left|P_{k}(K(\varphi))\right|$.

Received September 30, 1983. 
Though we have not yet obtained the generality including all of these theorems, we give a generalization of the first one in this paper. Let us denote the maximal unramified central extension of $K / k$ by $C(K / k)$. Then its genus field coincides with $\tilde{k}$. Therefore the degree $\left[C(K / k): S_{k}(K)\right]$ is a multiple of $\left|P_{k}(K)\right|=\left[\tilde{k}: S_{k}(K)\right]$. We show

Theorem 1. The degree $[K: k]$ divides $\left[C(K / k): S_{k}(K)\right]$.

Corollary. If $C(K / k)$ coincides with the genus field $\tilde{k}$, then $[K: k]$ divides $\left[\tilde{k}: S_{k}(K)\right]=\left|P_{k}(K)\right|$.

It is well known that every central extension of a cyclic extension coincides with its genus field. Therefore the corollary contains Hilbert's Theorem 94 as a special case.

We shall prove a stronger result. For an intermediate field $F$ of $K / k$, define the subfield $S_{F}(K)$ of $\tilde{F}$ as above for the unramified abelian extension $K / F$. Then it is not hard to see that $S_{F}(K)$ contains $S_{k}(K)$.

TheOREM 2. Let $F$ be a cyclic extension of $k$ of the maximal degree contained in $K$. Then $[K: k]$ divides

$$
\left[C(K / k) \cap S_{F}(K) \cdot K: S_{k}(K)\right] .
$$

A number theoretical description of the quotient will be given. (See Theorem 3 in $\S 2$ ).

As for the proofs, our basis is Artin [1], by which we reduce the things to group theoretic investigation of the transfers of the metabelian group $\operatorname{Gal}(\tilde{K} / k)$. The results are then also translated into theorems on the structure of the idele groups in Section 4 by the same way as in $[3]$.

\section{§2. The main theorem and its consequences}

Let $K / k$ be an unramified abelian extension of algebraic number fields. In addition to the notation given in the preceding section, let $\lambda_{K / k}: C \ell(k)$ $\rightarrow C \ell(K)$ be the homomorphism induced by lifting ideals of $k$ to the ones of $K$ naturally. Then $P_{k}(K)$ is the kernel of $\lambda_{K / k}$. We denote the homomorphism of $C \ell(K)$ to $C \ell(k)$ induced from the norm map of $K$ over $k$ by $N_{K / k}: C \ell(K) \rightarrow C \ell(k)$.

Let $F$ be an abelian extension of $k$ contained in $K$. The field $S_{F}(K)$ is the subfield of $\tilde{F}$ corresponding to $P_{F}(K)=\operatorname{Ker} \lambda_{K^{\prime} F}$ by class field 
theory. It is obvious by the definition that $N_{F / k}\left(P_{F}(K)\right) \subset P_{k}(K)$. Therefore we have

Proposition 1. $S_{k}(K) \subset S_{F}(K)$.

Proposition 2. Suppose that $F / k$ is a cyclic extension of the maximal degree contained in $K$. Then $\lambda_{F / k}(C \ell(k))$ is contained in $N_{K / F}(C \ell(K))$.

Proof. Let $c$ be an element of $\lambda_{F / k}(C \ell(k))$, and take $a \in C \ell(k)$ so that $c=\lambda_{F^{\prime} / k}(a)$. Then $N_{F / k}(c)=a^{[F: k]}$. By the choice of $F$, the degree $[F: k]$ coincides with the exponent of the abelian group $C \ell(k) / N_{K / k}(C \ell(K))$ which is isomorphic to Gal $(K / k)$. Therefore $N_{F / k}(c) \in N_{K / k}(C \ell(K))$. Take $b \in$ $C \ell(K)$ so that $N_{F / k}(c)=N_{K / k}(b)$. Then we have $c \cdot N_{K / F}(b)^{-1} \in \operatorname{Ker} N_{F / k}$. Since $K$ is contained in $\tilde{k} \cdot F=\tilde{k}$, we see that $N_{K / F}(C \ell(K))$ contains $\operatorname{Ker} N_{F / k}$. Therefore $c=\lambda_{F / k}(a)$ belongs to $N_{K / F}(C \ell(K))$ Q.E.D.

If $F / k$ satisfies the condition of the proposition, then $\lambda_{K / k}(C \ell(k))$ is contained in $\lambda_{K / F} \circ N_{K / F}(C \ell(K))$. Therefore it is a subgroup of

$$
\begin{aligned}
\left\{\lambda_{K / F} \circ\right. & \left.N_{K / F}(C \ell(K))\right\}^{\operatorname{Gal}(K / k)} \\
& \stackrel{\text { def }}{=}\left\{c \in \lambda_{K / F} \circ N_{K / F}(C \ell(K)) \mid c^{\sigma}=c \text { for } \forall_{\sigma} \in \operatorname{Gal}(K / k)\right\} .
\end{aligned}
$$

We now state our main theorem, the proof of which will be given in the next section.

Theorem 3. Let the notation and the assumptions be as above. Suppose that $F / k$ is a cyclic extension of the maximal degree contained in $K$. Then we have

$$
\begin{aligned}
& {\left[C(K / k) \cap S_{F}(K) \cdot K: S_{k}(K)\right]} \\
& \quad=[K: k] \cdot\left[\left\{\lambda_{K / F} \circ N_{K / F}(C \ell(K))\right\}^{\operatorname{Gal}(K / k)}: \lambda_{K / k}(C \ell(k))\right] .
\end{aligned}
$$

Corollary 1. Let the situation be as in the theorem. If $C(K / k) \cap$ $S_{F}(K) \subset \tilde{k}$, then $[K: k]$ divides $\left|P_{k}(K)\right|$.

Since $\left|P_{k}(K)\right|=\left[\tilde{k}: S_{k}(K)\right]$, this is obvious by the theorem. Theorems 1 and 2 in Section 1 are also immediate consequences of this theorem.

Corollary 2. Suppose that there exist subfields $F$ and $F^{\prime}$ of $K$ which satisfy the conditions (1) (3): (1) $F / k$ is a cyclic extension of the maximal degree contained in $K$; (2) $K=F \cdot F^{\prime}$ and $F \cap F^{\prime}=k$; (3) $\tilde{F} \cap \tilde{F}^{\prime}=\tilde{k}$. Then $[K: k]$ divides $\left|P_{k}(K)\right|$.

The proof will also be given in the next section. 


\section{§3. The proof of Theorem 3}

Let $K, k$ and $F$ be as in Theorem 3, and put $G=\operatorname{Gal}(\tilde{K} / k), A=$ $\operatorname{Gal}(\tilde{K} / K)$ and $H=\operatorname{Gal}(\tilde{K} / F)$. The commutator group $[G, G]$ of $G$ is equal to $\mathrm{Gal}(\tilde{K} / \tilde{k})$, and contained in $A$. By the choice of $F$, we see that $G / H$ is cyclic. Take $\xi \in G$ so that $G=\langle\xi\rangle \cdot H$. Note that $[F: k]$ is the exponent of the abelian group $G / A \cong \mathrm{Gal}(K / k)$. It follows from the definition that $\operatorname{Gal}(\tilde{K} / C(K / k))$ is equal to

$$
[G, A]=\left\langle g^{-1} a^{-1} g a \mid g \in G, a \in A\right\rangle .
$$

Let $V_{G \rightarrow A}: G \rightarrow A$ and $V_{H \rightarrow A}: H \rightarrow A$ be the transfers of $G$ and $H$ to the abelian subgroup $A$, respectively. They induce homomorphisms $\bar{V}_{G \rightarrow A}$ : $G /[G, G] \rightarrow A$ and $\bar{V}_{H \rightarrow A}: H /[H, H] \rightarrow A$. The transfer $V_{G \rightarrow H}: G \rightarrow H /[H, H]$ of $G$ to $H$ also induces a homomorphism $\bar{V}_{G \rightarrow H}: G /[G, G] \rightarrow H /[H, H]$. As is well known, we have $V_{G \rightarrow A}=\bar{V}_{H \rightarrow A} \circ V_{G \rightarrow H}$.

Denote the Artin maps of class field theory for $k, F$ and $K$ by $\alpha_{k}, \alpha_{F}$ and $\alpha_{K}$, respectively. They are isomorphisms of the following groups:

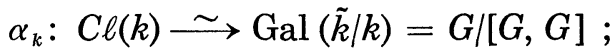

$$
\begin{aligned}
& \alpha_{F}: C \ell(F) \stackrel{\sim}{\longrightarrow} \operatorname{Gal}(\tilde{F} / F)=H /[H, H] ; \\
& \alpha_{K}: C \ell(K) \stackrel{\sim}{\longrightarrow} \operatorname{Gal}(\tilde{K} / K)=A .
\end{aligned}
$$

By Artin [1], we have the commutative diagram,

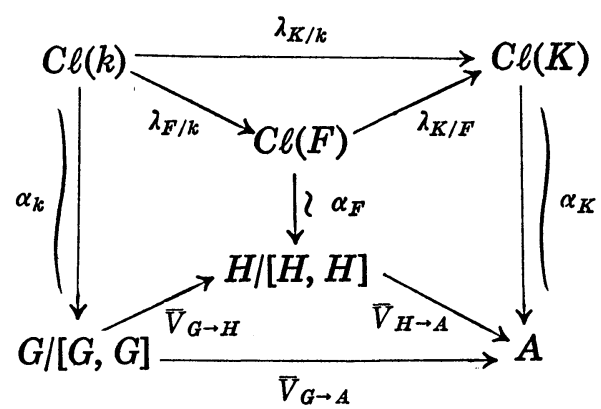

Therefore $\operatorname{Gal}\left(\tilde{K} / S_{k}(K)\right)=\operatorname{Ker} V_{G \rightarrow A}$ and $\operatorname{Gal}\left(\tilde{K} / S_{F}(K)\right)=\operatorname{Ker} V_{H \rightarrow A}$. Hence we have

LEMMA 1.

$\left[C(K / k) \cap S_{F}(K) \cdot K: S_{k}(K)\right]=\left[\operatorname{Ker} V_{G \rightarrow A}:[G, A] \cdot\left(A \cap \operatorname{Ker} V_{H \rightarrow A}\right)\right]$.

We also have 


$$
\alpha_{K} \circ \lambda_{K / k}(C \ell(k))=V_{G \rightarrow A}(G),
$$

and

$$
\alpha_{K} \circ \lambda_{K / F} \circ N_{K / F}(C \ell(K))=V_{I I \rightarrow A}(A)
$$

because $\alpha_{F} \circ N_{K / F} \circ \alpha_{K}^{-1}(a)=a \bmod [H, H]$ for $a \in A$, as is well known by class field theory. Since $A$ is a normal abelian subgroup of $G$, the action of $G$ on $A$ through inner automorphisms defines the structure of $(G / A)$ module on $A$. Then $\alpha_{K}$ is a $(G / A)$-isomorphism. Therefore we have

$$
\alpha_{K}\left(\left\{\lambda_{K / F} \circ N_{K / F}(C \ell(K))\right\}^{\operatorname{Gal}(K / k)}\right)=V_{H \rightarrow A}(A) \cap Z(G)
$$

where $Z(G)$ is the center of $G$, and the following lemma.

\section{LEMMA 2.}

$$
\left[\left\{\lambda_{K / F} \circ N_{K / F}(C \ell(K))\right\}^{\mathrm{Gal}(K / k)}: \lambda_{K / k}(C \ell(k))\right]=\left[V_{H \rightarrow A}(A) \cap Z(G): V_{G \rightarrow A}(G)\right] .
$$

For the completeness, let us show an elementary fact on transfers which we need.

Proposition 3. Let $\mathscr{B S}$ and $\mathfrak{F}_{1}$ be groups in general, $\mathfrak{S}$ a subgroup of (S) of finite index and $\varphi:$ \&S $\rightarrow \mathbb{S}_{1}$ a homomorphism. Suppose that $\operatorname{Ker} \varphi$ $\subset \mathfrak{S}$. Then we have $\bar{\varphi} \circ V_{\circledast \rightarrow \mathfrak{F}}=V_{\varphi(\Theta) \rightarrow \varphi(\mathfrak{F})} \circ \varphi$ where $\bar{\varphi}: \mathfrak{S E}_{\mathfrak{C}} /[\mathfrak{S E}, \mathfrak{S}] \rightarrow \varphi(\mathfrak{S}) /[\varphi(\mathfrak{S})$, $\varphi(\mathfrak{S})]$ is the homomorphism induced by $\varphi$.

Proof. Take a set of representatives $\left\{R_{i} \mid i=1, \cdots,\left[\&: \mathscr{S}_{]}\right]\right\}$of the cosets of $\mathbb{S} \bmod \mathfrak{S}$, i.e. $\mathscr{S}=\bigcup_{i} \mathfrak{S} \cdot R_{i}$ (disjoint). Since $\operatorname{Ker} \varphi \subset \mathfrak{F}$, we have $\varphi(\mathbb{S})=\bigcup_{i} \varphi(\mathfrak{S}) \cdot \varphi\left(R_{i}\right)$ (disjoint). Furthermore we see that $R_{i} \cdot G=H_{i}(G) \cdot R_{i}$, with $H_{i}(G) \in \mathfrak{S}$ if and only if $\varphi\left(R_{i}\right) \cdot \varphi(G)=\varphi\left(H_{i}(G)\right) \cdot \varphi\left(R_{i^{\prime}}\right)$ with $\phi\left(H_{i}(G)\right) \in$ $\varphi(\mathscr{S})$ for each $G \in \mathbb{S}$. Then we see the proposition at once by Huppert $[2,1.4, \mathrm{~b})]$.

COROLlaRY. Let (S) be a group and $\mathfrak{A}$ a normal abelian subgroup of (S) of finite index. Then $V_{\mathbb{G} \rightarrow \text { (S) }} \subset Z$ (ब) .

Proof. For $x \in \mathbb{S}$, let $\varphi$ : \&S $\widetilde{\rightarrow}$ (S) be the inner automorphism of (S) defined by $x$. Since $\mathfrak{A}$ is normal in $\mathbb{S}$ and abelian, we have, for $g \in \mathbb{S}$,

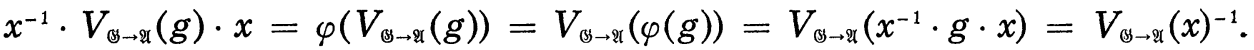
$V_{\mathscr{G} \rightarrow \mathfrak{A}}(g) \cdot V_{\mathscr{G} \rightarrow \mathfrak{A}}(x)=V_{\mathscr{G} \rightarrow \mathfrak{A}}(g)$. This is true for every $x \in \mathbb{S}$. Therefore, $V_{\Theta \rightarrow 2}(g)$ belongs to $Z(\circlearrowleft)$ Q.E.D.

It has already been proved as a part of Lemma 2 that $V_{G \rightarrow A}(G)$ lies in $V_{H \rightarrow A}(A) \cap Z(G)$. We have just shown group theoretically that $V_{G \rightarrow A}(G)$ 
$\subset Z(G)$. We also give a group theoretic proof to the fact that $V_{G \rightarrow A}(G)$ $\subset V_{H \rightarrow A}(A)$. This fact corresponds to Proposition 2 in the preceding section.

Proposition 4. Let $G \supset H \supset A$ be as above. Namely, $H$ and $A$ are normal subgroups of $G, A$ is abelian containing $[G, G]$, and $[G: H]$ coincides with the exponent of the abelian group $G / A$. Then $V_{G \rightarrow A}(G) \subset V_{H \rightarrow A}(A)$.

Proof. For $g \in G$, we have $V_{G \rightarrow A}(g)=\bar{V}_{H \rightarrow A} \circ V_{G \rightarrow H}(g)$. By Huppert $[2, I V, 1.7]$ for example, we easily see that $V_{G \rightarrow H}(g) \equiv g^{[G: H]}[H, H] \bmod [G, G]$. Since $[G: H]$ is the exponent of $G / A$, we see $V_{G \rightarrow H}(g) \in A /[H, H]$. Hence we have $V_{G \rightarrow A}(g) \in V_{H \rightarrow A}(A)=\bar{V}_{H \rightarrow A}(A /[H, H])$.

Q.E.D.

Let us continue the proof of Theorem 3. Put

$$
q=\frac{\left[\operatorname{Ker} V_{G \rightarrow A}:[G, A] \cdot\left(A \cap \operatorname{Ker} V_{H \rightarrow A}\right)\right]}{[G: A] \cdot\left[V_{H \rightarrow A}(A) \cap Z(G): V_{G \rightarrow A}(G)\right]} .
$$

Then by Lemmas 1 and 2 , it is sufficient to show that $q=1$ since $[K: k]$ $=[G: A]$. Multiplying both of the numerator and the denominator of $q$ by $\left|V_{G \rightarrow A}(G)\right|=\left[G\right.$ : $\left.\operatorname{Ker} V_{G \rightarrow A}\right]$, we have

$$
\begin{aligned}
q & =\frac{\left[G:[G, A] \cdot\left(A \cap \operatorname{Ker} V_{H \rightarrow A}\right)\right]}{[G: A] \cdot\left|V_{H \rightarrow A}(A) \cap Z(G)\right|} \\
& =\frac{\left[A:[G, A] \cdot\left(A \cap \operatorname{Ker} V_{H \rightarrow A}\right)\right]}{\left|V_{H \rightarrow A}(A) \cap Z(G)\right|} .
\end{aligned}
$$

Since $G=\langle\xi\rangle \cdot H$ and $V_{H \rightarrow A}(A) \subset Z(H)$, we have $V_{H \rightarrow A}(A) \cap Z(G)=V_{H \rightarrow A}(A)$ $\cap C_{A}(\xi)$ where $C_{A}(\xi)$ is the centralizer of $\xi$ in $A$.

Lemma 3. The map $\varphi: A \rightarrow A$ defined by $\varphi(a)=[\xi, a]=\xi^{-1} a^{-1} \xi a$ for $a \in A$ is an endomorphism of $A$ with $\operatorname{Ker} \varphi=C_{A}(\xi)$.

Proof. For $a, b \in A$, we have

$$
\begin{aligned}
{[\xi, a \cdot b] } & =[\xi, b] \cdot[\xi, a]^{b} \\
& =[\xi, b] \cdot[\xi, a] \cdot[[\xi, a], b] .
\end{aligned}
$$

Since $A$ is normal in $G$ and abelian, we have $[[\xi, a], b]=1$, and

$$
[\xi, a \cdot b]=[\xi, a] \cdot[\xi, b] \text {. }
$$

This shows that $\varphi: A \rightarrow A$ is a well defined homomorphism. It is obvious that $\operatorname{Ker} \varphi=C_{A}(\xi)$.

Q.E.D. 
Lemma 4. $[G, A]=[\xi, A] \cdot[H, A]=\varphi(A) \cdot[H, A]$.

Proof. For $x, y \in G$ and $a \in A$, we have

$$
\begin{aligned}
{[x \cdot y, a] } & =[x, a]^{y} \cdot[y, a] \\
& =[x, a] \cdot[[x, a], y] \cdot[y, a] \\
& =[x, a] \cdot[y,[x, a]]^{-1} \cdot[y, a] \\
& =[x, a] \cdot\left[y,[x, a]^{-1} \cdot a\right]
\end{aligned}
$$

because $A$ is normal in $G$ and abelian. Since $G=\langle\xi\rangle \cdot H$, we have the desired result.

Put $\psi=\left.V_{H \rightarrow A}\right|_{A}: A \rightarrow A$. Then this is an endomorphism of $A$ with $\operatorname{Ker} \psi=A \cap \operatorname{Ker} V_{H \rightarrow A}$. Since $\operatorname{Ker} \psi$ contains $[H, A]$, we have

$$
\begin{aligned}
q & =\frac{[A: \operatorname{Im} \varphi \cdot \operatorname{Ker} \psi]}{|\operatorname{Im} \psi \cap \operatorname{Ker} \varphi|} \\
& =\frac{[A: \operatorname{Im} \varphi]}{|\operatorname{Im} \psi \cap \operatorname{Ker} \varphi| \cdot[\operatorname{Im} \varphi \cdot \operatorname{Ker} \psi: \operatorname{Im} \varphi]} .
\end{aligned}
$$

Since $[A: \operatorname{Im} \varphi]=|\operatorname{Ker} \varphi|$ and $[\operatorname{Ker} \varphi: \operatorname{Im} \psi \cap \operatorname{Ker} \varphi]=[\operatorname{Im} \psi \cdot \operatorname{Ker} \varphi: \operatorname{Im} \psi]$, we finally obtain

$$
q=\frac{[\operatorname{Im} \psi \cdot \operatorname{Ker} \varphi: \operatorname{Im} \psi]}{[\operatorname{Im} \varphi \cdot \operatorname{Ker} \psi: \operatorname{Im} \varphi]}
$$

Lemma 5. We have $\varphi \circ \psi=\psi \circ \varphi$. Therefore $q=1$.

Proof. For $a \in A$, we have $(\varphi \circ \psi)(a)=\left[\xi, V_{H \rightarrow A}(a)\right]=\xi^{-1} \cdot V_{H \rightarrow A}\left(a^{-1}\right) \cdot \xi$. $V_{H \rightarrow A}(a)$. Since $H$ is a normal subgroup of $G$, the inner automorphism of $G$ defined by $\xi$ induces an automorphism of $H$, which maps $A$ onto itself. Therefore we have $\xi^{-1} \cdot V_{H \rightarrow A}\left(a^{-1}\right) \cdot \xi=V_{H \rightarrow A}\left(\xi^{-1} a^{-1} \xi\right)$ by Proposition 3 for $\mathbb{S}=H$ and $\mathfrak{S}=A$. Hence we have

$$
\begin{aligned}
(\varphi \circ \psi)(a) & =V_{H \rightarrow A}\left(\xi^{-1} a^{-1} \xi\right) \cdot V_{H \rightarrow A}(a) \\
& =V_{H \rightarrow A}\left(\xi^{-1} a^{-1} \xi a\right)=(\psi \circ \varphi)(a) .
\end{aligned}
$$

Thus we have shown that $\varphi \circ \psi=\psi \circ \varphi$.

Now put $B=\operatorname{Im}(\varphi \circ \psi)=\operatorname{Im}(\psi \circ \varphi)$. Then $\varphi(B) \subset B$ and $\psi(B) \subset B$. Therefore $\varphi$ and $\psi$ induce endomorphisms of $\bar{A}=A / B$, which we denote by $\bar{\varphi}$ and $\bar{\psi}$ respectively. Then $\bar{\varphi} \circ \bar{\psi}=\bar{\psi} \circ \bar{\varphi}=$ trivial. By Herbrand's lemma (see Huppert [2, III, 19.4]), we have

$[\operatorname{Ker} \bar{\varphi}: \operatorname{Im} \bar{\psi}]=[\operatorname{Ker} \bar{\psi}: \operatorname{Im} \bar{\varphi}]$. 
Let us show that $\operatorname{Ker} \bar{\varphi}=(\operatorname{Ker} \varphi \cdot \operatorname{Im} \psi) / B$. In fact, suppose that $\varphi(a) \in B$ for $a \in A$. Take $b \in A$ so that $\varphi(a)=\varphi(\psi(b))$. Then $a \cdot \psi(b)^{-1} \in \operatorname{Ker} \varphi$. Therefore $a=\left(a \cdot \psi(b)^{-1}\right) \cdot \psi(b) \in \operatorname{Ker} \varphi \cdot \operatorname{Im} \psi$. It is obvious that $\varphi$ maps $\operatorname{Ker} \varphi \cdot \operatorname{Im} \psi$ into $B$. Thus we have $\operatorname{Ker} \bar{\varphi}=(\operatorname{Ker} \varphi \cdot \operatorname{Im} \psi) / B$. By the same way, we also have $\operatorname{Ker} \bar{\psi}=(\operatorname{Ker} \psi \cdot \operatorname{Im} \varphi) / B$. Since both of $\operatorname{Im} \varphi$ and $\operatorname{Im} \psi$ contain $B$, we have $q=1$ by the above equality.

Q.E.D.

The proof of Theorem 3 is also completed.

Proof of Corollary 2 to Theorem 3. Suppose that $F$ and $F^{\prime}$ are given as in the corollary. Using the same notation as above, we may assume that $H=\operatorname{Gal}(\tilde{K} / F)$ and $\langle\xi\rangle \cdot A=\operatorname{Gal}\left(\tilde{K} / F^{\prime}\right)$. Then $[H, H]=\operatorname{Gal}(\tilde{K} / \tilde{F})$ and $[\langle\xi\rangle A,\langle\xi\rangle A]=\operatorname{Gal}\left(\tilde{K} \mid \tilde{F}^{\prime}\right)$. Since $A$ is abelian, we have $[\langle\xi\rangle A,\langle\xi\rangle A]$ $=[\xi, A]$. Therefore $\operatorname{Gal}\left(\tilde{K} / \tilde{F} \cap \tilde{F}^{\prime}\right)=[\xi, A] \cdot[H, H]$. By the assumption (3), we have $[G, G]=[\xi, A] \cdot[H, H]$. Since $\operatorname{Ker} V_{H \rightarrow A}$ contains $[H, H]$, we see $[G, G]$ lie in $[G, A] \cdot\left(A \cap \operatorname{Ker} V_{H \rightarrow A}\right)=\operatorname{Gal}\left(\tilde{K} / C(K / k) \cap S_{F}(K) \cdot K\right)$. This shows that $\tilde{k}$ contains $C(K / k) \cap S_{F}(K) \cdot K$. Therefore Corollary 2 follows from Corollary 1 to Theorem 3 . The proof is completed.

\section{$\S 4$. The adelic version}

Let $k_{A}^{\times}$be the idele group of $k, k_{\infty+}^{\times}$the connected component of the unity of the Archimedian part of $k_{A}^{\times}$and $k^{\#}$ the closure of $k^{\times} \cdot k_{\infty+}^{\times}$in $k_{A}^{\times}$. Let $K$ be an abelian extension of $k$ of finite degree. (K/k is not necessarily unramified.) Put $\mathrm{g}=\mathrm{Gal}(K / k)$, and let $K_{A}^{\Delta_{\mathrm{g}}}$ be the closed subgroup of the idele group $K_{A}^{\times}$of $K$ defined by

$$
K_{A}^{\Delta_{\mathfrak{g}}}=\left\langle x^{1-\sigma} \mid x \in K_{A}^{\times}, \sigma \in \mathfrak{g}\right\rangle .
$$

Let $N_{K / k}: K_{A}^{\times} \rightarrow k_{A}^{\times}$be the norm map. We consider $k_{A}^{\times}$a subgroup of $K_{A}^{\times}$ naturally.

Suppose that we are given a subfield $F$ of $K$ such that $F$ is cyclic over $k$ of the maximal degree. The idele group $F_{A}^{\times}$is also considered a subgroup of $K_{A}^{\times}$. Let $N_{K / F}: K_{A}^{\times} \rightarrow F_{A}^{\times}$be the norm map of $K$ over $F$.

THEOREM 4. Let the notation and the assumptions be as above. Let $U$ be an open subgroup of $K_{A}^{\times}$, and suppose that $U \supset K^{\times} \cdot K_{\infty+}^{\times}$and that $U^{\sigma}=U$ for each $\sigma \in \mathrm{g}$. Put

$$
\left\{N_{K / F}\left(K_{A}^{\times}\right) \cdot U / U\right\}^{\mathfrak{g}}=\left\{c \in N_{K / F}\left(K_{A}^{\times}\right) \cdot U / U \mid c^{\sigma}=c \text { for } \forall \sigma \in \mathfrak{g}\right\} .
$$

Then we have 


$$
\begin{aligned}
& {\left[k_{A}^{\times} \cap U: k^{\times} \cdot N_{K / k}\left(K_{A}^{\times}\right) \cap U\right] \cdot\left[N_{K / k}^{-1}\left(k_{A}^{\times} \cap U\right): K_{A}^{\Delta_{A}} \cdot N_{K / F}^{-1}\left(F_{A}^{\times} \cap U\right)\right]} \\
& =[K: k] \cdot\left[\left\{N_{K / F}\left(K_{A}^{\times}\right) \cdot U / U\right\}^{g}: k_{A}^{\times} \cdot U / U\right] .
\end{aligned}
$$

Proof. Let $k_{\mathrm{ab}}, K_{\mathrm{ab}}$ and $F_{\mathrm{ab}}$ be the maximal abelian extensions of $k$, $K$ and $F$, respectively, in the algebraic closure of $k$. The Artin maps of $k, K$ and $F$ are open continuous surjective homomorphisms

$$
\begin{aligned}
& \alpha_{k}: k_{\boldsymbol{A}}^{\times} \longrightarrow \operatorname{Gal}\left(k_{\mathrm{ab}} / k\right), \\
& \alpha_{K}: K_{\boldsymbol{A}}^{\times} \longrightarrow \operatorname{Gal}\left(K_{\mathrm{ab}} / K\right),
\end{aligned}
$$

and

$$
\alpha_{F}: F_{\boldsymbol{A}}^{\times} \longrightarrow \operatorname{Gal}\left(F_{\mathrm{ab}} / F\right),
$$

respectively, the kernels of which are $k^{\sharp}, K^{\sharp}$ and $F^{\sharp}$. Let $\bar{K}$ be the subfield of $K_{\mathrm{ab}}$ corresponding to the open subgroup $\alpha_{K}(U)$ of $\mathrm{Gal}\left(K_{\mathrm{ab}} / K\right)$. Then $\bar{K}$ is normal over $k$. Put $G=\operatorname{Gal}(\bar{K} / k), A=\operatorname{Gal}(\bar{K} / K)$ and $H=\operatorname{Gal}(\bar{K} / F)$. Then $A$ and $H$ are normal in $G$. Furthermore $A$ is abelian and contains $[G, G]$. We have the following commutative diagram whose three columns are exact:

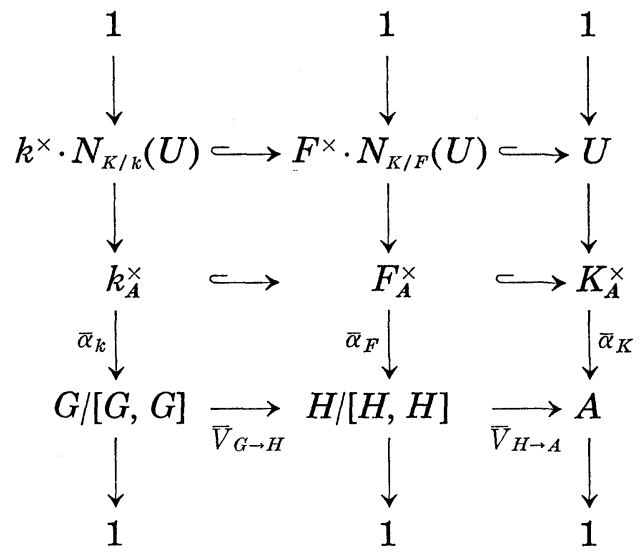

Here $\bar{\alpha}_{k}, \bar{\alpha}_{F}$ and $\bar{\alpha}_{K}$ are the homomorphisms naturally induced from $\alpha_{k}$, $\alpha_{F}$ and $\alpha_{K}$, respectively. (Cf. [3, Proposition 3] for example.) Therefore we have homomorphisms

$$
\begin{aligned}
& \bar{\alpha}_{k}: k_{\boldsymbol{A}}^{\times} \cap U / k^{\times} \cdot N_{K / k}(U) \stackrel{\sim}{\longrightarrow} \operatorname{Ker} V_{G \rightarrow A} /[G, G], \\
& \bar{\alpha}_{F}: F_{\boldsymbol{A}}^{\times} \cap U / F^{\times} \cdot N_{K / F^{\prime}}(U) \stackrel{\sim}{\longrightarrow} \operatorname{Ker} V_{H \rightarrow A} /[H, H] .
\end{aligned}
$$

Furthermore, we have, for $x \in K_{A}^{\times}$, 


$$
\bar{\alpha}_{F} \circ N_{K / F}(x)=\bar{\alpha}_{K}(x) \cdot[H, H], \quad \text { and } \quad \bar{\alpha}_{F}\left(N_{K / F}\left(K_{A}^{\times}\right)\right)=A /[H, H]
$$

by class field theory. This shows that

$$
V_{H \rightarrow A}(A)=\bar{\alpha}_{K}\left(N_{K / F}\left(K_{A}^{\times}\right)\right) \simeq N_{K / F}\left(K_{A}^{\times}\right) \cdot U / U .
$$

Note that these isomorphisms are ones of $\mathrm{g}$-modules for $\mathrm{g}=\mathrm{Gal}(K / k)=$ $G / A$.

Let us now interpret the equality

$$
q=\frac{\left[\operatorname{Ker} V_{G \rightarrow A}:[G, A] \cdot\left(A \cap \operatorname{Ker} V_{H \rightarrow A}\right)\right]}{[G: A] \cdot\left[V_{H \rightarrow A}(A) \cap Z(G): V_{G \rightarrow A}(G)\right]}=1
$$

which was proved in the previous section. In the similar way there, we have $[G: A]=[K: k]$ and

$$
\left[V_{H \rightarrow A}(A) \cap Z(G): V_{G \rightarrow A}(G)\right]=\left[\left\{N_{K / F}\left(K_{A}^{\times}\right) \cdot U / U\right\}^{g}: k_{A}^{\times} \cdot U / U\right]
$$

in the present situation. As for the numerator, it is equal to

$\left[\operatorname{Ker} V_{G \rightarrow A}: A \cap \operatorname{Ker} V_{G \rightarrow A}\right] \cdot\left[A \cap \operatorname{Ker} V_{G \rightarrow A}:[G, A] \cdot\left(A \cap \operatorname{Ker} V_{H \rightarrow A}\right)\right]$.

We have

$$
\begin{aligned}
& {\left[\operatorname{Ker} V_{G \rightarrow A}: A \cap \operatorname{Ker} V_{G \rightarrow A}\right]} \\
& \quad=\left[\operatorname{Ker} V_{G \rightarrow A} /[G, G]:\left(A \cap \operatorname{Ker} V_{G \rightarrow A}\right) /[G, G]\right] \\
& \quad=\left[k_{A}^{\times} \cap U: k^{\times} \cdot N_{K / k}\left(K_{A}^{\times}\right) \cap U\right]
\end{aligned}
$$

because the subgroup $A /[G, G]$ of $G /[G, G]$ is equal to $\bar{\alpha}_{k}\left(N_{K / k}\left(K_{A}^{\times}\right)\right)$. Furthermore, we also have

$$
\bar{\alpha}_{K}\left(N_{K / k}^{-1}\left(k_{A}^{\times} \cap U\right)\right)=A \cap \operatorname{Ker} V_{G \rightarrow A},
$$

and

$$
\bar{\alpha}_{K}\left(N_{K / F}^{-1}\left(F_{A}^{\times} \cap U\right)\right)=A \cap \operatorname{Ker} V_{H \rightarrow A}
$$

because, by class field theory, the following diagrams are commutative:
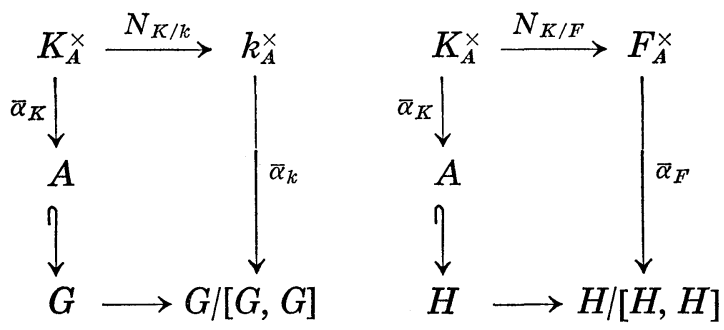
where the homomorphisms of the last row are the natural projections. Since $\bar{\alpha}_{K}\left(K_{A}^{\lrcorner_{g}}\right)=[G, A]$ and $N_{K / F}^{-1}\left(F_{A}^{\times} \cap U\right) \supset U=\operatorname{Ker} \bar{\alpha}_{K}$, we finally have

$$
\begin{aligned}
& {\left[A \cap \operatorname{Ker} V_{G \rightarrow A}:[G, A] \cdot\left(A \cap \operatorname{Ker} V_{H \rightarrow A}\right)\right]} \\
& \quad=\left[N_{\bar{K} / k}^{-1}\left(k_{A}^{\times} \cap U\right): K_{A}^{\Delta_{A}} \cdot N_{\bar{K} / k}^{-1}\left(F_{A}^{\times} \cap U\right)\right] .
\end{aligned}
$$

The equality, $q=1$, now gives the equality of the theorem at once.

Corollary. Let $K / k$ be an abelian extension of finite degree with $\mathfrak{g}=$ $\mathrm{Gal}(K / k)$. Let $U$ be an open subgroup of $K_{A}^{\times}$which contains $K^{\times} \cdot K_{\infty_{+}}^{\times}$and satisfies that $U^{\sigma}=U$ for each $\sigma \in \mathfrak{g}$. If $U \cdot K_{A}^{\Delta_{\mathrm{g}}}$ contains $N_{K / k}^{-1}\left(k^{\sharp}\right)$, then $[K: k]$ divides $\left[k_{\boldsymbol{A}}^{\times} \cap U: k^{\times} \cdot N_{K / k}(U)\right]$.

Proof. We have $k^{\times} \cdot N_{K / k}\left(K_{A}^{\times}\right)=k^{\#} \cdot N_{K / k}\left(K_{A}^{\times}\right)$and $k^{\times} \cdot N_{K / k}(U)=$ $k^{\sharp} \cdot N_{K / k}(U)$. If, therefore, $U \cdot K_{A}^{\Delta_{8}}$ contains $N_{K / k}^{-1}\left(k^{\sharp}\right)$, we have

$$
\begin{aligned}
& {\left[N_{K / k}^{-1}\left(k_{A}^{\times} \cap U\right): K_{A}^{\Delta_{\beta}} \cdot U\right]} \\
& \quad=\left[k^{\times} \cdot N_{K / k}\left(K_{A}^{\times}\right) \cap U: k^{\times} \cdot N_{K / k}(U)\right] .
\end{aligned}
$$

Therefore $\left[k_{A}^{\times} \cap U: k^{\times} \cdot N_{K / k}\left(K_{A}^{\times}\right) \cap U\right] \cdot\left[N_{K / k}^{-1}\left(k_{A}^{\times} \cap U\right): K_{A}^{\Delta_{A}} \cdot U\right]$ is equal to $\left[k_{A}^{\times} \cap U: k^{\times} \cdot N_{K / k}(U)\right]$. Since $K_{A}^{\Delta_{\beta}} \cdot U$ is a subgroup of $K_{A}^{\Delta_{\mathrm{g}}} \cdot N_{K / k}^{-1}\left(F_{A}^{\times} \cap U\right)$, we have the corollary from the theorem at once.

Remark 1. If $K / k$ is unramified and $U=O^{\times}\left(K_{A}\right)=$ the unit group of the adele ring $K_{A}$, then Theorem 4 is equivalent to Theorem 3 , and the corollary to the one to Theorem 1 in Section 1 .

Remark 2. Let $L$ be the abelian extension of $K$ corresponding to $U$ in the corollary. Then the maximal central extension $L^{*}$ of $K / k$ contained in $L$ corresponds to $U \cdot K_{A}^{\Delta_{g}}$. Therefore the condition, $U \cdot K_{A}^{\Delta_{g}} \supset N_{K / k}^{-1}\left(k^{\sharp}\right)$, is equivalent to the one that $L^{*}$ is contained in $K \cdot k_{\mathrm{ab}}$, i.e. that $L^{*}$ reduces to its genus field $L \cap K \cdot k_{\mathrm{ab}}$.

\section{REFERENCES}

[1] E. Artin, Idealklassen in Oberkörpern und allgemeine Reziprozitätsgesetze, Abh. Math. Sem. Univ. Hamburg., 7 (1930), 46-51.

[2] B. Huppert, Endliche Gruppen I, Springer-Verlag, Berlin·Heidelberg $\cdot$ New York (1967).

[3] K. Miyake, On the structure of the idele groups of algebraic number fields, II, Tôhoku Math. J., 34 (1982), 101-112. 
Department of Mathematics College of General Education Nagoya University

Chikusa-ku, Nagoya 464

Japan 